\title{
Le FMI à la rescousse : la zone euro a-t-elle bénéficié de l'expérience du fonds dans la lutte contre les crises?
}

Barbara Fritz, Sebastian Dullien et Laurissa Mühlich

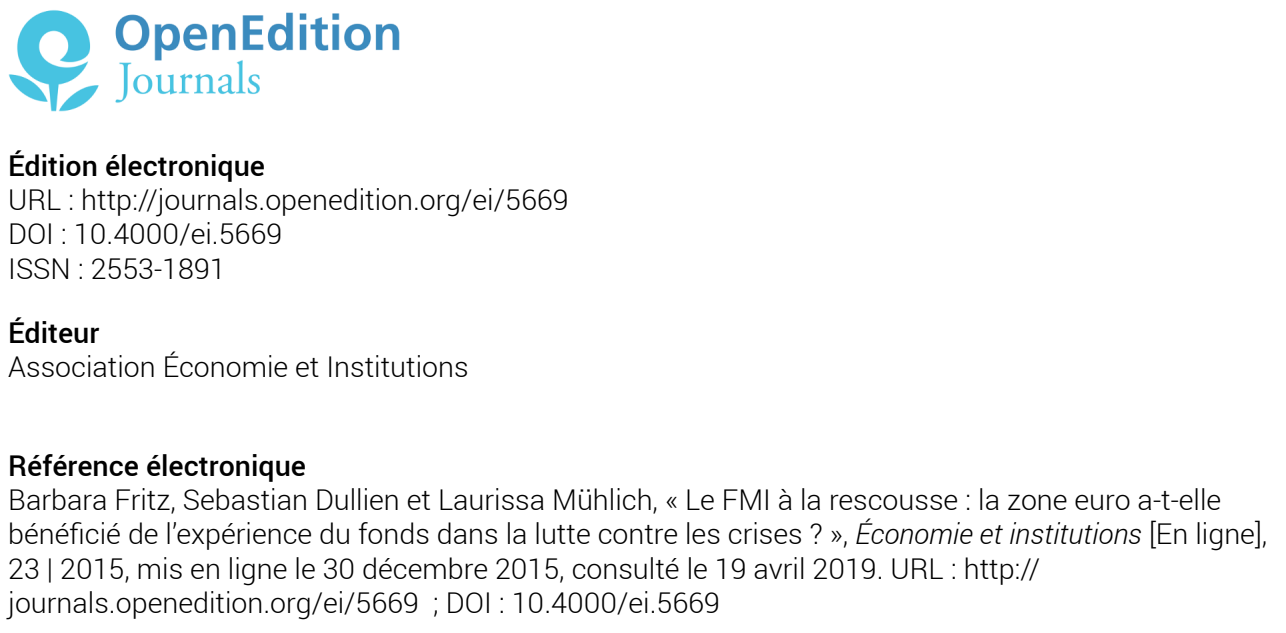

Ce document a été généré automatiquement le 19 avril 2019

Revue Économie et institutions 


\title{
Le FMI à la rescousse : la zone euro a-t-elle bénéficié de l'expérience $d u$ fonds dans la lutte contre les crises?
}

\author{
Barbara Fritz, Sebastian Dullien et Laurissa Mühlich
}

\section{Introduction}

1 La crise de l'euro a apporté des changements considérables: non seulement l'Union monétaire européenne a opéré une refonte de sa structure de gouvernance et centralisé la supervision du système bancaire, mais cette crise a également marqué un tournant majeur pour le système monétaire global. Après avoir perdu la plupart de ses emprunteurs habituels, le FMI a endossé un nouveau rôle à la suite de la crise de 2008-2009: il lui a été demandé, d'une part, de mettre sur pied de nombreux plans de sauvetage de grande ampleur, et il a été sollicité, d'autre part, pour soutenir des économies avancées en Europe pour la première fois depuis 1977.

En outre, la participation du FMI aux plans de sauvetage en Grèce, au Portugal et en Irlande a été très particulière en raison des attentes des pays emprunteurs: pour les Européens, c'était moins la nécessité d'obtenir des fonds qui les ont conduit à demander au FMI de participer à l'organisation de la troïka (également composée de la Commission européenne et de la Banque centrale européenne, ou BCE), que la volonté de tirer parti de l'expérience significative du FMI dans la conception et la mise en œuvre de plans de sauvetage et de programmes d'ajustement en temps de crise (Pisani-Ferry et al., 2013).

Dans les milieux politiques, la décision a été vigoureusement défendue et perçue comme une réussite. C'est ainsi que le formula l'ancien directeur de la BCE, Jörg Asmussen : « Il s'est avéré juste d'inclure le FMI. Le Fonds possède une expérience unique dans la 
conception de ce type de programmes. Il s'agit en outre d'une sorte de gendarme externe à l'Europe capable d'agir avec moins de d'états d'âme » (Asmussen, 201212).

Dans cet article, nous nous demanderons de quelle manière le FMI a apporté l'expérience unique qu'il a acquise dans l'assistance aux économies moins avancées. Pour répondre à cette question, nous analyserons trois aspects. Premièrement, au vu des connaissances théoriques sur les crises financières telles que celle de la zone euro, quelle aurait été la conception idéale du mode de résolution? Deuxièmement, dans quelle mesure le FMI a-til intégré cette conception dans les programmes destinés aux marchés émergents à la fin des années 1990 et au début des années 2000 et comment les orientations du FMI furentelles révisées? Troisièmement, dans quelle mesure le FMI a-t-il réussi à transférer cette expérience dans la gestion de la crise de l'euro? Nous analyserons ces questions en dégageant les idées essentielles de cette institution concernant la gestion des crises de liquidité et de solvabilité.

5 Ces idées ont évolué. On peut identifier trois générations de publications sur les crises économiques. On interrogera ce savoir au regard des cadres réglementaires du FMI et des politiques mises en place dans les économies émergentes et en Europe. Nous n'aborderons pas les transformations institutionnelles ou les processus de prise de décision au sein du FMI qui auraient pu contribuer à ces changements de politique.

6 La suite de cet article est organisée comme suit. Dans la première partie, nous examinerons brièvement l'évolution des modèles théoriques sur les crises financières et ferons une synthèse des prescriptions optimales pouvant être tirées de ces modèles en ce qui concerne les prêts de sauvetage et les programmes d'ajustement. Dans la seconde partie, nous comparerons ces changements avec la politique du FMI dans les crises des économies émergentes les plus remarquables de ces deux dernières décennies, à savoir la crise argentine de 2001-2002 et la crise brésilienne de 2002. Nous examinerons également les enseignements que le FMI a directement tirés de ces expériences. Enfin, dans la dernière partie, nous comparerons ces nouvelles directives et l'approche de la troïka face aux pays de la zone euro.

\section{Problèmes de liquidités vs problèmes de solvabilité : trois générations de théorisation des crises financières}

7 Depuis la chute du système de Bretton Woods dans les années 1970, les turbulences accrues sur les marchés financiers ont suscité un intérêt soutenu pour la théorisation des crises financières dont découleront, dans les années 1980 et 1990, plusieurs générations de modèles portant sur les crises de balance des paiements. Le débat reste ouvert quant à la nature des crises (budgétaire, bancaire, compétitivité, etc.) qui ont interagi dans la zone euro (Shambaugh, 2012, Fratzscher, 2013). De même, la classification des crises des économies émergentes passées est d'un intérêt majeur (Kaminsky et Reinhart, 1999 ou Laeven et Valencia, 2012). Au regard de ces débats, on peut dégager certaines leçons à tirer de ces modèles afin de préciser quels en sont les aspects réellement pertinents dans le cas de la crise de la zone euro.

8 Les modèles de crise de la balance des paiements se fondent sur l'idée selon laquelle, d'une part, un actif donné n'est plus disponible qu'en quantité limitée (qu'il s'agisse des réserves de change en cas de pure crise de la balance des paiements ou des recettes du 
gouvernement dans le cas des crises de la dette souveraine) et, d'autre part, le stock du dit actif tend à s'épuiser, que ce soit en raison d'erreurs de politique économique ou d'une fuite des investisseurs, ou encore de la combinaison des deux.

9 La première génération de modèles explique les attaques contre des monnaies à taux de change fixe par des anticipations rationnelles pessimistes, dues à des politiques gouvernementales incohérentes ou à des fuites du marché d'obligations publiques qui, l'une ou l'autre, rendent la dette publique insoutenable. Par exemple, lorsqu'un déficit budgétaire fait monter l'inflation intérieure au-dessus du niveau international, les sorties de capitaux vont obliger l'autorité monétaire à abandonner le taux de change fixe (Krugman, 1979). Curieusement, la fuite des capitaux a lieu dans ce cas avant même que les réserves de change ne soient épuisées, les investisseurs estimant que les pertes de réserves ne peuvent être enrayées.

Transposé à la question de la dette souveraine, ce modèle peut expliquer qu'une politique budgétaire mettant un pays dans une position dans laquelle il sera incapable à l'avenir de rembourser sa dette pousserait les principaux investisseurs à interrompre le financement des déficits budgétaires ; contraignant par conséquent un gouvernement au défaut. Dans cette situation, étant donné que l'origine du défaut est une politique budgétaire insoutenable, un programme d'ajustement requiert un changement des politiques existantes vers ce qui est appelé de façon générique l'austérité, à savoir une combinaison de coupes des dépenses et une augmentation des recettes. En fait, cet ajustement est l'aspect le plus important de la lutte contre la crise, dans la mesure où il est considéré comme seul moyen pour remettre les pays sur une trajectoire viable. En outre, si aucune voie d'ajustement n'est assurée de réussir, parce que le volume de la dette est déjà trop important, l'ajustement doit alors être mis en œuvre conjointement avec une restructuration de la dette. Des prêts de trésorerie peuvent être octroyés pour limiter les répercussions sur l'économie réelle.

11 La mécanique est différente dans les modèles de seconde génération (Obstfeld, 1996). Ces derniers ne supposent pas nécessairement une défaillance claire en matière de politique économique comme point de départ. Tandis que les pays conduisant une politique monétaire ou budgétaire clairement insoutenable vont se retrouver en crise comme dans les modèles de première génération, les modèles de seconde génération incluent par ailleurs la possibilité d'équilibres multiples pour les pays menant des politiques économiques qui ne sont pas nettement insoutenables.

Comme l'ont montré Cole et Kehoe (1996), cette configuration mène à la possibilité de crises de la dette ou de crises budgétaires auto-réalisatrices. La logique est simple : pour une entité avec un niveau de dette modéré (qui n'est pas encore extrêmement élevé), la possibilité de rembourser cette dette dépend des attentes des acteurs du marché. Si les investisseurs pensent qu'un pays endetté est capable de répondre à leurs exigences, ils acceptent des taux d'intérêt plus bas et la dette peut donc être soutenable. Dans le cas d'un débiteur souverain, si les investisseurs pensent que l'État en question n'est pas capable de rembourser sa dette, ils exigent des taux d'intérêt plus élevés et la dette devient insoutenable, menant par conséquent au défaut.

Dans ces modèles, étant donné qu'un changement dans les prévisions peut provoquer une crise, même sans modification des principes de base sous-jacents, il est difficile de déterminer la raison spécifique du déclenchement d'une crise (Krugman, 1999). 

continu à l'emprunt à des taux d'intérêt raisonnables, les prévisions vont se stabiliser durablement sur un "bon » équilibre et une crise budgétaire auto-réalisatrice n'est par conséquent plus possible. L'action de cette tierce partie aiderait à éviter des coûts considérables pour l'économie en cas de succès de la prévision d'une crise. La nécessité de programmes d'ajustement macroéconomiques est beaucoup moins évidente dans le cadre d'une crise auto-réalisatrice que dans le cadre d'une crise standard des modèles de première génération : évidemment, un ratio dette/PIB plus bas semble rendre les crises moins probables. Cependant, comme ce sont les prévisions qui ont déclenché la crise, il n'y a pas de condition nécessaire à la résolution de la crise. Comme dans certains cas, l'austérité peut effectivement faire baisser le PIB de telle sorte que le niveau du ratio dette/PIB augmente (Hollande et Portes, 2012). Par conséquent, ces programmes doivent être conçus avec précaution. Corsetti, et al., 1998, Krugman, 2003) ont par la suite exploré la manière dont les problèmes du système bancaire et financier interagissent avec les crises de la dette publique et les crises monétaires, ainsi que la manière dont ils se renforcent mutuellement, soulignant la pertinence d'une prise de décision rapide et efficace pour empêcher que ces crises ne se propagent à l'économie réelle et dans d'autres pays.

Empêcher qu'une crise auto-réalisatrice se produise engage deux grandes préoccupations quant à la fourniture de liquidités par un acteur tiers extérieur, telle que mentionnée plus haut: premièrement, la tierce partie doit être disponible et capable de fournir une quantité suffisante de liquidités pour infléchir les prévisions des marchés; deuxièmement, elle doit faire une distinction claire entre une situation d'illiquidité et une situation d'insolvabilité.

17 En ce qui concerne le premier point, la fourniture de liquidités, dans le cas d'un système bancaire national avec des contrats financiers libellés en monnaie nationale, il existe généralement une banque centrale habilitée à agir en tant que prêteur en dernier ressort (PDR) et qui peut donc émettre sans limites des liquidités en monnaie nationale. La logique d'un PDR pour assurer la stabilité monétaire et financière au niveau national est connue depuis les travaux précurseurs de Bagehot sur l'émergence de la Banque d'Angleterre en tant que première banque centrale du monde moderne (Bagehot, 1873).

Néanmoins, l'approvisionnement en liquidités est moins simple tant pour le cas d'une dette libellée en devise étrangère que pour celui d'une dette publique, notamment en ce qui concerne la zone euro. Premièrement, dans le cas d'une dette extérieure libellée en devise étrangère, la banque centrale nationale ne peut pas faire office de PDR, puisqu'elle ne peut pas en émettre. Le principal accès au financement hors marché est la fourniture de liquidités par le FMI, étant donné qu'il n'existe pas de PDR au niveau mondial (Eichengreen et Hausmann, 2005). Deuxièmement, pour tout cas d'un débiteur public sous pression, il y a généralement désaccord sur la question de savoir si une banque centrale doit agir en tant que PDR, ou si cela nuit à sa mission principale de maintenir la stabilité des prix et génère un aléa moral qui pousserait les gouvernements à dépasser leurs budgets et à attendre à un renflouement de la banque centrale. De manière générale, les principes directeurs des banques centrales tendent à s'éloigner du seul objectif de stabilité des prix pour cibler la stabilité financière dans son ensemble (Blanchard et al., 2010 ; Eichengreen et al., 2011). Cependant, la question de savoir si cela englobe les efforts clairement définis visant à préserver la liquidité des gouvernements nationaux pour 
rétablir le «bon équilibre » est un objet de litige largement débattu même si les attentes prédominantes du marché peuvent indiquer autre chose (Eichengreen et al., 2011, p. 24 ; voir également Blanchard, 2012). Finalement, pour la zone euro, la fourniture de liquidités aux gouvernements nationaux par la $\mathrm{BCE}$ est restreinte par les règles des traités de l'Union européenne qui interdisent l'achat direct d'obligations d'État, tout comme la fourniture de facilités de découvert. À cet égard, l'encours de la dette des gouvernements nationaux de la zone euro a les caractéristiques d'une dette libellée en devise étrangère.

Deuxièmement, il est essentiel que l'acteur tiers fasse la distinction entre les problèmes de liquidités et de solvabilité. Les entités insolvables sont définies comme étant incapables s'acquitter de leurs obligations à moyen terme et à long terme, même si on les approvisionne en liquidités supplémentaires à court terme. Fournir des liquidités à une entité insolvable signifie par conséquent que l'ajournement de la résolution de l'insolvabilité conduit à des coûts plus élevés.

20 Pour les pays confrontés à des crises de liquidités, il est également nécessaire de savoir si la panique des investisseurs a été provoquée par des mesures qui ont rapproché le pays d'une trajectoire budgétaire insoutenable ou si la panique a plutôt été déclenchée par un événement étranger à la politique budgétaire. Un solide ajustement de la politique budgétaire est souhaitable et nécessaire uniquement dans le premier cas.

\section{Comment le FMI a introduit la distinction entre crise de liquidités et crise de solvabilité ?}

21 Ces évolutions théoriques n'ont pas été sans incidence sur le FMI. Bien au contraire, les crises des économies émergentes des années 1990 ont exposé à de vives critiques la gestion qu'il en a faites (e. g. Stiglitz, 2002) et il est devenu de plus en plus manifeste pour le Fonds qu'il faisait face à un triple défi, théorique, de gestion et financier. Il y a répondu par une révision de ses principales approches et politiques.

D'un point de vue théorique, le fait que la plupart de ces crises monétaires soient survenues dans des contextes de réformes favorables aux marchés, tout particulièrement de libéralisation des échanges et de libéralisation financière, a soulevé un défi spécifique. Il était assez évident que celles-ci ne pouvaient être aisément justifiées comme étant les conséquences de purs échecs politiques, conformément aux modèles de crise monétaire de première génération, mentionnés plus haut, alors que les crises monétaires et les crises de la dette dans les pays en développement pendant les années 1980 avaient été traitées en tant que conséquences des stratégies de substitution des importations. Dans le nouveau contexte de la libéralisation, les économistes du FMI se sont rapidement mis à réfléchir à la pertinence des modèles d'équilibres multiples pour ce nouveau type de crise. Par exemple, dans un document de travail du FMI, Flood et Marion (1996) ont établi un lien direct entre ces modèles et la crise mexicaine du peso en 1994 (voir également Masson, 1999). Ces réflexions ne concernaient pas seulement les questions liées aux taux de change, mais également la question des conditions dans lesquelles le FMI devait agir en tant qu'acteur tiers en injectant des liquidités pour rétablir le «bon équilibre » dans les limites des attentes du marché.

Sans attendre, suite à son intervention en Argentine, sur laquelle il avait essuyé des critiques cinglantes (voir ci-avant le point 3.1), le FMI a commencé à développer un plan sans équivoque pour distinguer les entités illiquides des entités insolvables qui peuvent 
être objet d'intervention dans les crises du compte de capital ou dans les crises monétaires. Ce plan a pris en compte les connaissances théoriques nouvelles, débattues plus haut. Dans un document programmatique basé sur le "Processus de Prague" (Köhler, 2000), le FMI a établi une nouvelle ligne directrice par laquelle il mettait en avant la distinction suivante : les crises de liquidités devraient être résolues par une fourniture de liquidités rapide et suffisante, alors que les problèmes de solvabilité devraient être affrontés par une restructuration de la dette, laquelle doit non seulement prendre en compte la prolongation des échéances, mais également une décote réduisant la valeur nominale de la dette.

Dans une directive institutionnelle ultérieure intitulée «Assessing sustainability (FMI, 2002a), le FMI a livré une définition opérationnelle des concepts de liquidités et de solvabilité :

«Une entité est solvable si la valeur actualisée (present discounted value - PDV) de ses dépenses primaires courantes et futures n'est pas plus élevée que la valeur actualisée de l'évolution de ses revenus courants et futurs, nets de tout endettement initial. [...] Une entité est illiquide si, indépendamment de savoir si elle remplit les conditions de la solvabilité, ses actifs liquides et ses financements disponibles sont insuffisants pour rembourser ou refinancer ses dettes arrivant à échéance (FMI, 2002a, p. 5).»

Dans le même document, le FMI a admis que la distinction entre crise de liquidité et crise de solvabilité n'allait pas de soi. Premièrement car ces concepts sont vagues : à moins d'être résolue immédiatement, une crise de liquidité conduira à un accroissement des coûts de financement et donc à une augmentation de la valeur actualisée de la dette. Par conséquent, le FMI a commencé à mettre en œuvre le concept de soutenabilité de la dette, qui signifie la conformité à la fois avec les critères de liquidités et ceux de solvabilité : « La soutenabilité englobe ainsi les concepts de solvabilité et de liquidités, sans établir de nette démarcation entre les deux » (FMI, 2002a, p. 4). Deuxièmement, il admet en outre que ce dernier concept était loin d'être simple à appréhender empiriquement. La valeur actualisée de la dette et le service de la dette sont principalement déterminés par des paramètres macroéconomiques comme le taux d'intérêt et le taux de croissance, et - dans le cas des économies émergentes - le taux de change, qui par définition est endogène aux prévisions du marché quant à la solvabilité et aux liquidités du débiteur. Le FMI a conclu que «les estimations de soutenabilité sont ainsi intrinsèquement probabilistes et qu'aucune action ne peut s'esquiver de la nécessité de statuer » (FMI, 2002a, p. 6).

En outre, en raison de la dérégulation financière, les flux de capitaux internationaux privés ont fortement augmenté jusqu'au début des années 2000. Ainsi, le quota appliqué par le FMI jusqu'alors (basé sur la quote-part des pays au FMI) pour déterminer le volume de liquidités qu'un pays pouvait recevoir de l'institution a été considéré comme très insuffisant. À la fin de l'année 2002, le FMI a de ce fait instauré la "politique d'accès exceptionnel » (Exceptional Access Policy - EAP), devant être appliquée à : « tout prêt pour lequel l'accès dépasse $100 \%$ du quota sur une base annuelle ou au-dessus de $300 \%$ de quota cumulatif indépendamment du mécanisme utilisé » (FMI, 2003, p. 5).

Sur la base des considérations liées aux questions de liquidité et de solvabilité, cet accès à des fonds importants a été associé à des règles exceptionnellement strictes (FMI, 2003, p. 3f) : premièrement, il doit uniquement concerner des cas de pressions exceptionnelles de la balance des paiements; deuxièmement, et c'est le plus important, «[une] analyse rigoureuse et systématique [devrait établir] une forte probabilité que la dette reste soutenable » (FMI, 2003, p. 4, souligné par les auteurs) ; troisièmement, le pays doit avoir de bonnes 
chances d'accéder à nouveau aux marchés des capitaux privés; et quatrièmement, la fourniture de liquidités doit être soutenue par une solide conception de sa programmation et de sa mise en œuvre dans le cadre des politiques d'ajustement.

Le programme de crédit accordé au Brésil en 2002 a été plutôt couronné de succès et il fut ainsi considéré comme une preuve du bien-fondé de l'augmentation de la capacité de prêt potentiel du FMI. En 2006, au cours d'un séminaire intitulé "Who needs the IMF?", Kenneth Rogoff qualifia de tournant majeur l'assistance fournie par le FMI au Brésil en 2002:

«Prenez par exemple l'enveloppe de prêts, aussi risquée que créative, octroyée par le FMI au Brésil en 2002 (...). Dans une situation où l'accès aux marchés est gelé et le pays au bord du défaut, le Fonds intervint avec 30 milliards d'euros. Le prêt du Fonds aida sans doute à éviter un effondrement qui aurait démoli les marchés mondiaux, de Manille à Istanbul, et il a anticipé la période favorable dont les économies émergentes ont profité ces dernières années (Rogoff, 2006).»

Les enseignements tirés par le FMI après des expériences de crises peuvent être ainsi résumés en trois points: premièrement, évaluer si le problème est de l'ordre de la liquidité ou de la solvabilité, les prêts ne pouvant être attribués que dans le premier cas ; deuxièmement, si tel est bien le cas, les dispositifs de veille devraient suffire amplement à dissiper quelque doute que ce soit sur leur montant approprié ; et troisièmement, exiger une politique d'ajustement uniquement selon son degré de nécessité pour retrouver l'accès aux marchés.

Une analyse historique des principes et directives du Fonds montre que depuis sa fondation, « les concepts, les cadres analytiques, les techniques, et les méthodologies que le personnel du Fonds a développés au fil des années ont presque toujours évolué suite à un problème pratique immédiat auquel un de ses membres a fait face ». (De Vries, 1987, p. 16).

31 Sur la base des documents du FMI concernant les cas de l'Argentine et du Brésil, nous affirmons tout d'abord que les expériences évoquées dans cette section prennent leur source dans les crises argentines du début des années 2000 et l'implication très critiquée du Fonds (voir partie 2.1). Deuxièmement, nous interprétons le cas de la provision rapide de liquidités au Brésil en 2002 (partie 2.2) comme une application immédiate des connaissances nouvellement acquises. D'autres auteurs confirment que le FMI n'est plus dans les années 2010 ce qu'il était auparavant (Ban et Gallagher, 2015); nous identifions ce changement à ces épisodes.

\subsection{L'Argentine}

Le FMI a été impliqué en Argentine depuis le milieu des années 1990 et il s'est vu luimême mis en cause dans l'aggravation de la crise à la fin des années 1990. Après la crise, la réponse que le FMI lui avait apportée a été considérée comme hautement problématique, à la fois par le Fonds lui-même et par les observateurs externes. En conséquence, l'intervention du FMI en Argentine a conduit à une réflexion approfondie et à une redéfinition postérieure de ses politiques.

33 Le fond de la crise argentine résidait dans son régime de caisse d'émission à taux de change fixe que le gouvernement avait mis en place au début des années 1990 afin de combattre l'inflation. Ainsi, la banque centrale argentine était uniquement autorisée à émettre de la monnaie en contrepartie des réserves de change. Par conséquent, elle 
n'était pas capable de mener une politique anticyclique et n'était pas en position d'agir en tant que PDR dans le cas où ses banques faisaient face à des problèmes de liquidités.

Tandis que ce système avait réussi à faire retomber l'inflation sous la barre des deux chiffres au début des années 1990, et qu'il avait amélioré la confiance des investisseurs et ainsi rouvert la voie au retour des afflux de capitaux dans la première partie de la décennie, le pays se retrouve à nouveau confronté à une importante fuite de capitaux dès le début de la crise asiatique. Celle-ci se déclenche dans un contexte où un différentiel d'inflation permanent avec les États-Unis avait sapé la compétitivité de l'Argentine. Ce problème a été exacerbé par la dévaluation au Brésil, premier partenaire commercial du pays. La banque centrale ne pouvant pas créer de monnaie, la fuite de capitaux a conduit à une grave contraction du crédit.

À partir de 1999, l'économie argentine ne cesse de se contracter jusqu'à atteindre en 2002 une croissance négative du PIB de $-10,9 \%$ (voir tableau 1), perdant sur cette période environ un quart de sa capacité de production. Ce fut la plus grave crise économique dont un pays (hors économies en transition) ait souffert depuis la Grande Dépression. La combinaison entre récession et baisse des recettes fiscales a mené à une forte hausse du ratio dette/PIB. Début 2002, le pays a déclaré le défaut de sa dette extérieure. Les discussions sur la restructuration de la dette avec les créanciers privés n'ont été menées à leur terme qu'en 2005, quand la majorité des détenteurs d'obligations a accepté le report des échéances et la réduction de la valeur nominale de la dette ${ }^{2}$.

Entre 1996 et 2001, le FMI avait conclu trois accords de financement avec l'Argentine, un premier dans le cadre du Mécanisme élargi de crédit (MEDC) en 1998 (classé "préventif » - aucun tirage ne fut d'ailleurs effectué) et ensuite deux Accords de confirmation (Standby arrangement ou SBA, 1999 et 2000). Début 2000, le FMI affichait l'intention de s'occuper d'une situation économique qui se détériorait au point d'entraver l'accès du pays aux marchés financiers internationaux et il octroie un SBA d'environ 7,2 milliards de dollars. Ce plan incluait une austérité drastique: début 2000, le FMI et l'Argentine se sont entendus sur le fait que le gouvernement devait faire passer le budget fédéral d'un déficit de 2,5\% du PIB à l'équilibre en « 2003 au plus tard » (FMI, 2000a).

Le SBA a été relevé de 13,7 milliards de dollars début 2001 tandis que l'austérité était intensifiée à travers le protocole d'accord de décembre 2000, qui annonçait désormais une augmentation de quatre points de l'excédent primaire consolidé du secteur public entre 2001 et 2005 (FMI, 2000b). En outre, le FMI a piloté un plan de financement international de créanciers publics et privés, publicisé sous le terme de blindaje (bouclier) et qui s'élevait à 39 milliards de dollars américains (FMI, 2004a, p. 9). Au même moment, dans une tentative ultime et désespérée, le gouvernement argentin a mis en œuvre une vaste restructuration des obligations publiques nationales qui a prolongé les échéances des obligations et devait réduire les coûts publics de refinancement. Cependant, les taux d'intérêt se sont accrus malgré tout et par conséquent les coûts de refinancement n'ont pas baissé. Au contraire, les perspectives d'un défaut proche se sont précisées et la fuite des capitaux s'est donc poursuivie. À l'automne 2001, le SBA mentionné plus haut a été de nouveau augmenté d'environ 22 milliards de dollars, dont trois milliards étaient supposés soutenir une possible intervention de restructuration de la dette.

Le plan de sauvetage n'a donc pas redonné accès aux marchés financiers internationaux; le retour des afflux de capitaux ne s'est pas produit. Les contraintes de paiement du pays sont devenues énormes, à la mesure des primes de risques croissantes sur les crédits internationaux. Celles-ci ont augmenté tout au long de l'année 2001, passant d'un niveau 
déjà élevé de 1000 points de base à environ 5000. Finalement, au terme de l'année 2001, le FMI a interrompu son financement de l'Argentine pour cause de non-respect du programme budgétaire convenu et de manque de confiance des marchés (FMI, 2004a, p. 9).

Quelques voix au sein du FMI ont continué à soutenir que le problème majeur avait été la politique budgétaire insoutenable de l'Argentine, tout particulièrement Michael Mussa, chef du département de recherche du FMI jusqu'en 2001 (Mussa, 2002, p. 10ff). Si cela avait été avéré, un ajustement de ces politiques aurait pu mettre fin à la crise et les prêts du FMI auraient pu être considérés comme une provision raisonnable et transitoire pour faciliter le processus d'ajustement.

Néanmoins, d'autres diagnostics de la crise mettaient en avant des problèmes plus fondamentaux. Un examen de la composition des rentrées fiscales et des dépenses budgétaires pendant la période de la crise a montré que la forte hausse des ratios dette publique/PIB (voir tableau 1) était principalement due à l'augmentation du remboursement de la dette publique et à la contraction du PIB, tandis que le solde primaire, c'est à dire le solde budgétaire avant paiements d'intérêts, témoignait d'un léger excédent de $0,45 \%$ en moyenne pendant cette période. Les intérêts de la dette publique ont augmenté de 10,9 \% en 1997 à 23,4 \% en 2001 (Damill et al., 2012b, p. 9).

41 Malgré un solide ajustement du solde primaire du secteur public, le cercle vertueux n'a jamais été atteint. Pire, l'augmentation des impôts et des coupes dans les dépenses publiques ont renforcé la tendance récessive, alimentant ainsi les perspectives négatives qui empêchaient que se réalise la chute attendue de la prime de risque du pays.

42 La seule politique budgétaire était donc impuissante pour compenser les importants déséquilibres macroéconomiques, plus particulièrement en ce qui concerne les comptes extérieurs (Damill et al., 2012a, p. 4).

Le ratio dette publique/PIB n'était pas excessivement élevé mais la dette publique était presque entièrement libellée en devise étrangère et une dépréciation en aurait fortement augmenté le fardeau. En même temps, l'Argentine était incapable de générer de la croissance économique, vu le taux de change (surévalué) de l'époque. Autrement dit: dans le cadre macroéconomique existant, l'Argentine devait être considérée comme insolvable.

44 Avec le recul, le FMI est arrivé aux mêmes conclusions, semble-t-il. Il s'est en tous cas démarqué de sa position antérieure. Flemming Larsen, alors directeur des bureaux Europe du FMI, déclarait en 2003 : «Le FMI aurait dû maintenir sa conclusion de 1998 selon laquelle, d'une part, le régime à taux de change fixe était insoutenable et d'autre part, les autorités semblaient également réticentes ou incapables d'ajuster suffisamment leur politique pour éviter un éventuel effondrement » (Larsen, 2003). Néanmoins, il tient les autorités nationales pour responsables du prolongement des programmes du FMI sur cette trajectoire insoutenable: "Ces inquiétudes [quant à un possible effondrement financier] ont été exprimées à maintes reprises mais les autorités ont refusé d'envisager une sortie du système de caisse d'émission jusqu'à ce qu'il y soit contraint par les marchés » (Larsen, 2003).

La rigidité du système de caisse d'émission posait indéniablement un problème majeur, celui d'impliquer une totale absence de prévision d'une possibilité de sortie harmonieuse. Certes, le FMI ne pouvait être tenu pour responsable de l'introduction de ce régime en Argentine au début des années 1990, mais il faut rappeler que, plus tard, il a 
vigoureusement prôné cette version inflexible d'un taux de change fixe pour stabiliser les prévisions du marché dans le cadre des équilibres multiples. Cet ancrage rigide du taux de change avait - entre autres - produit une forte appréciation du taux de change réel, soutenue par des afflux de capitaux considérables dans la période d'avant-crise. Par la suite, le pays avait cumulé une aggravation du déficit de la balance courante et une hausse de la dette extérieure en devise étrangère. L'une et l'autre sont devenues insoutenables en même temps que cette évolution rendait le changement du régime monétaire impossible en pratique, sans un défaut de la dette.

Quoi qu'il en soit, même si les autorités nationales ont insisté pour maintenir le régime monétaire, il était de la responsabilité du FMI de stopper de nouveaux prêts à un pays clairement insolvable. La suite d'offres de liquidités qu'a octroyées le FMI dans les années précédant le défaut et la restructuration de la dette se basaient sur l'hypothèse que le pays entretenait une politique budgétaire instable à laquelle il était encore possible, en principe, de remédier par des coupes budgétaires. Une telle hypothèse était manifestement contradictoire avec son évaluation précédente d'une insoutenabilité de la dette du pays, reconnue au moins à huis clos.

Tableau 1. Argentine : Indicateurs économiques sélectionnés

\begin{tabular}{|l|l|l|l|l|l|l|l|l|}
\hline & $\mathbf{1 9 9 8}$ & $\mathbf{1 9 9 9}$ & $\mathbf{2 0 0 0}$ & $\mathbf{2 0 0 1}$ & $\mathbf{2 0 0 2}$ & $\mathbf{2 0 0 3}$ & $\mathbf{2 0 0 4}$ \\
\hline Croissance du PIB (\% annuel) (1) & 3,85 & $-3,39$ & $-0,79$ & $-4,41$ & $-10,89$ & 8,84 & 9,03 \\
\hline $\begin{array}{l}\text { Montant de la dette extérieure (\% du } \\
\text { PNB) (1) }\end{array}$ & 48,06 & 54,34 & 53,15 & 57,37 & 153,84 & 132,46 & 114,36 \\
\hline $\begin{array}{l}\text { Dette nette des administrations publiques } \\
\text { (\% du PB) (2) }\end{array}$ & n/a & n/a & n/a & n/a & n/a & n/a & n/a \\
\hline $\begin{array}{l}\text { Dette brute des administrations publiques } \\
\text { (\% du PIB) (2) }\end{array}$ & 38,18 & 43,49 & 45,62 & 53,62 & 164,99 & 139,45 & 127,03 \\
\hline $\begin{array}{l}\text { Solde structurel des administrations } \\
\text { publiques (\% du PIB potentiel) (2) }\end{array}$ & $-3,57$ & $-4,85$ & $-4,53$ & $-6,13$ & $-11,85$ & $-2,94$ & $-2,42$ \\
\hline $\begin{array}{l}\text { Taux de change officiel (UML par dollar US, } \\
\text { période moyenne) (1) }\end{array}$ & 1,00 & 1,00 & 1,00 & 1,00 & 3,06 & 2,90 & 2,92 \\
\hline $\begin{array}{l}\text { Taux d'intérêts réel (\%) (1) } \\
\text { Solde des transactions courantes (\% du PIB) } \\
(2)\end{array}$ & $-4,84$ & $-4,20$ & $-3,15$ & $-1,41$ & 9,00 & 6,38 & 1,75 \\
\hline $\begin{array}{l}\text { Inflation, parité de pouvoir d'achat (\% du } \\
\text { change) (2) }\end{array}$ & 0,93 & $-1,17$ & $-0,94$ & $-1,07$ & 25,87 & 13,44 & 4,42 \\
\hline
\end{tabular}

SOURCE: (1) WORLD DEVELOPMENT INDICATORS, 2013; (2) WORLD ECONOMIC OUTLOOK, AVRIL 2014. 


\subsection{Le Brésil}

côté, depuis la dernière crise financière en 1999 et l'abandon du régime d'ancrage du taux de change ayant donné lieu à une très forte dévaluation, la croissance du PIB s'était rétablie en 2000. Par ailleurs, tant le niveau de la dette extérieure totale (privée et publique) que celui de la dette publique (nationale et internationale) était relativement modéré. L'encours de la dette extérieure variait autour de $40 \%$ du PNB et la dette publique était inférieure à $50 \%$ du PIB en 2001 (voir tableau 2). Au même moment, la dette publique présentait toutefois une extrême vulnérabilité due à son indexation au taux de change et au fait qu'une grande partie était libellée en devise. Par la suite, avec les fuites de capitaux et une dépréciation de la monnaie nationale de presque $50 \%$, le ratio dette publique/PIB s'est donc fortement accru.

«La dépréciation du taux de change du fait de la crise de confiance lors de la campagne présidentielle en octobre a conduit à une hausse du ratio dette/PIB en conséquence de la réévaluation des obligations indexées au taux de change et des en-cours libellés en devise. Pour cette raison, et à cause du resserrement monétaire concomitant, les dépenses de paiement des intérêts ont considérablement augmenté, ce qui a mené à une détérioration du solde budgétaire global en 2002-2003, malgré le maintien d'un excédent primaire robuste (DeMello et Moccero, 2006, p. 13)."

51 Le FMI n'a pas classé la situation comme un cas d'insolvabilité mais plutôt comme un cas d'illiquidité temporaire, avec un risque associé qu'il se transforme rapidement en insolvabilité si la défiance du marché persistait. En juillet 2002, le FMI est intervenu avec un prêt sous forme d'Accord de confirmation de 30,4 milliards de dollars (22,8 milliards de DTS), courant sur un période de 15 mois - à l'époque, il s'agissait du plus haut niveau d'aide jamais atteint en termes de Droits de tirage spéciaux (DTS) (voir également FMI 
[2002b]). Comme ce montant dépassait de loin le quota normal du Brésil qui était de 3,04 milliards de DTS, de facto, c'était donc là un test de l'efficacité de l'EAP qui venait d'être mise en place en septembre 2002, lequel deviendra pleinement opérationnel en février 2003 (FMI, 2003, p. 3). Contrairement à d'autres programmes, ce dernier n'exigeait pas d'austérité drastique supplémentaire : dans le Protocole d'accord de juin 2002 à août 2002, l'objectif d'excédent primaire dans le budget de 2002 et 2003 a juste été légèrement augmenté de 3,5\% du PIB à 3,75\% du PIB, reflétant une contraction minime par rapport aux 3,6 \% atteints en 2001 (FMI, 2002a, 2002b).

Le cas du Brésil démontre les difficultés d'une évaluation appropriée d'une situation d'illiquidité avec une forte probabilité de soutenabilité de la dette. Barry Eichengreen, qui avait été conseillé politique pour le FMI les années précédentes, a plaidé publiquement en faveur de l'institution, alors fortement critiquée :

«Le Brésil de 2002 représente un meilleur pari pour le FMI que ne l'était l'Argentine de 2001. C'est l'occasion pour le Fonds de démontrer qu'il aide les économies bien gérées comme le Brésil tout en n'appliquant l'adage «qui aime bien qui châtie bien » qu'aux délinquants comme l'Argentine » (Eichengreen, 2002, p. 3).

L'auteur précisait par ailleurs que si le risque d'un nouveau crédit était élevé, celui de laisser les besoins financiers du Brésil sans surveillance était encore plus élevé, pouvant déboucher sur une situation d'insolvabilité. En outre, l'auteur a suggéré que ce n'était pas envisageable pour des raisons géopolitiques : «Pour sûr, si le pari est perdu, la chute sera rude. Mais parfois parier a plus de sens que ne rien parier du tout, malgré le risque de perdre. » (Eichengreen, 2002, p. 6)

La majeure partie du montant de l'Accord de confirmation (24 milliards de dollars) n'aura été déboursée qu'après les élections présidentielles. En même temps, malgré ses projets de transformation du modèle économique du pays, le candidat de gauche Lula s'était personnellement engagé - condition informelle préalable à l'Accord de confirmation dans une « Lettre au peuple brésilien » à " respecter tous les contrats et les obligations du pays " (Silva, 2002, p. 3)3. En conséquence, en seulement deux mois et avant même les élections présidentielles, les flux de capitaux étaient revenus et le taux de change brésilien avait presque retrouvé son niveau d'origine. ${ }^{4}$

Tableau $n^{\circ} 2$ : Brésil : Indicateurs économiques sélectionnés

\begin{tabular}{|l|l|l|l|l|l|l|l|}
\hline & $\mathbf{1 9 9 9}$ & $\mathbf{2 0 0 0}$ & $\mathbf{2 0 0 1}$ & $\mathbf{2 0 0 2}$ & $\mathbf{2 0 0 3}$ & $\mathbf{2 0 0 4}$ & $\mathbf{2 0 0 5}$ \\
\hline Croissance du PIB (\% annuel) (1) & 0,26 & 4,31 & 1,31 & 2,66 & 1,15 & 5,71 & 3,16 \\
\hline $\begin{array}{l}\text { Montant de la dette extérieure (\% du PNB) } \\
(1)\end{array}$ & 43,15 & 38,68 & 43,04 & 47,68 & 44,13 & 34,28 & 21,98 \\
\hline $\begin{array}{l}\text { Dette publique intérieure nette (\% du PIB) } \\
(2)\end{array}$ & 35,16 & 36,54 & 38,85 & 37,48 & 41,66 & 40,18 & 44,13 \\
\hline $\begin{array}{l}\text { Dette publique extérieure nette } \text { (\% du PIB) } \\
(2)\end{array}$ & 9,38 & 9,00 & 9,59 & 12,99 & 10,69 & 6,82 & 2,33 \\
\hline $\begin{array}{l}\text { Dette nette des administrations publiques (\% } \\
\text { du PIB) (3) }\end{array}$ & 41,76 & 42,83 & 47,54 & 49,24 & 51,67 & 47,21 & 46,38 \\
\hline
\end{tabular}




\begin{tabular}{|l|l|l|l|l|l|l|l|l|}
\hline $\begin{array}{l}\text { Dette brute des administrations publiques } \\
(\% \text { du PIB) (3) }\end{array}$ & 53,54 & 60,31 & 64,93 & 64,89 & 70,44 & 65,41 & 67,38 \\
\hline $\begin{array}{l}\text { Dette nette des administrations publiques ( \% } \\
\text { du PIB) (4) }\end{array}$ & n/a & 47,75 & 52,03 & 60,38 & 54,83 & 50,61 & 48,44 \\
\hline $\begin{array}{l}\text { Dette brute des administrations publiques } \\
\text { (\% du PIB) (4) }\end{array}$ & n/a & 66,65 & 70,79 & 79,38 & 74,61 & 70,66 & 69,33 \\
\hline $\begin{array}{l}\text { Taux de change officiel (UML par dollar US, } \\
\text { période moyenne (1) }\end{array}$ & 1,81 & 1,83 & 2,35 & 2,92 & 3,08 & 2,93 & 2,43 \\
\hline Taux SELIC (\% par an, fin de l'année) (3) & 19,04 & 15,84 & 19,05 & 24,90 & 16,33 & 17,75 & 18,04 \\
\hline $\begin{array}{l}\text { Inflation, parité de pouvoir d'achat (\% du } \\
\text { change) (2) }\end{array}$ & 4,86 & 7,04 & 6,84 & 8,45 & 14,71 & 6,60 & 6,87 \\
\hline $\begin{array}{l}\text { Solde des transactions courantes (\% du PIB) } \\
(2)\end{array}$ & $-4,317$ & $-3,757$ & $-4,189$ & $-1,509$ & 0,756 & 1,76 & 1,585 \\
\hline
\end{tabular}

\section{Comment la troïka a fait face à la crise de l'euro}

Force est de constater que ces expériences n'ont pas toutes été prises en compte dans la gestion de la crise de l'euro. Bien au contraire, celle-ci peut être considérée comme un exemple où, en premier lieu, on a fourni des liquidités à la Grèce sans restructuration de la dette bien qu'il était clair que le pays était insolvable. Par la suite, une austérité drastique a été imposée à des pays qui avaient avant tout des problèmes de liquidité, provoqués par la panique des investisseurs et les incertitudes du marché.

Au début de la crise de l'euro, les finances des gouvernements de nombreux pays de la zone euro furent mises à rude épreuve du fait des effets négatifs de la crise économique et financière mondiale de 2008/2009. En moyenne, la zone euro affichait un déficit public de $6,3 \%$ en 2009 , tous les pays ayant dépassé le seuil de $3 \%$ du PIB du traité de Maastricht. Néanmoins, à ce moment, la situation des finances publiques ne semblait pas trop préoccupante pour la plupart des États membres. L'Irlande et l'Espagne tout particulièrement (plus tard étiquetés "pays en crise»), ainsi que le Portugal, ne présentaient pas alors un niveau de ratio dette publique/PIB excessivement élevé.

Tableau 3

\begin{tabular}{|l|l|l|l|l|}
\hline \multicolumn{1}{|l|}{} & \multicolumn{2}{l|}{$\begin{array}{l}\text { Déficit public en \% du } \\
\text { PIB }\end{array}$} & $\begin{array}{l}\text { Dette publique en \% du } \\
\text { PIB }\end{array}$ \\
\hline Pays & 2008 & 2009 & 2008 & 2009 \\
\hline Zone euro (12 pays) & $-2,1$ & $-6,3$ & 70,9 & 80,7 \\
\hline Belgique & $-1,1$ & $-5,6$ & 89,2 & 95,7 \\
\hline
\end{tabular}




\begin{tabular}{|l|l|l|l|l|}
\hline Allemagne & $-0,1$ & $-3,1$ & 66,8 & 74,5 \\
\hline Irlande & $-7,4$ & $-13,7$ & 44,2 & 64,4 \\
\hline Grèce & $-9,9$ & $-15,6$ & 112,9 & 129,7 \\
\hline Espagne & $-4,5$ & $-11,1$ & 40,2 & 54,0 \\
\hline France & $-3,3$ & $-7,5$ & 68,2 & 79,2 \\
\hline Italie & $-2,7$ & $-5,4$ & 106,1 & 116,4 \\
\hline Chypre & 0,9 & $-6,1$ & 48,9 & 58,5 \\
\hline Luxembourg & 3,2 & $-0,7$ & 14,4 & 15,5 \\
\hline Malte & $-4,6$ & $-3,7$ & 60,9 & 66,5 \\
\hline Pays-Bas & 0,5 & $-5,6$ & 58,5 & 60,8 \\
\hline Autriche & $-1,0$ & $-4,1$ & 63,8 & 69,2 \\
\hline Portugal & $-3,7$ & $-10,2$ & 71,7 & 83,7 \\
\hline Finlande & 4,3 & $-2,7$ & 33,9 & 43,5 \\
\hline Royaume-Uni & $-5,0$ & $-11,3$ & 51,9 & 67,1 \\
\hline États-Unis & $-7,0$ & $-12,7$ & 73,3 & 86,3 \\
\hline Japon & $-8,8$ & 191,8 & 210,2 \\
\hline
\end{tabular}

Source : Ameco database (2014)

\subsection{Au sujet de la Grèce}

57 La crise de l'euro a commencé début 2010 quand le gouvernement grec nouvellement élu s'est vu contraint de réviser à la hausse les chiffres de son déficit et de sa dette. Selon les chiffres mis à jour, le déficit public en 2009 a été finalement réévalué à plus de $10 \%$ du PIB. Étant donné le niveau déjà élevé de la dette publique grecque, plus de $120 \%$ du PIB, les acteurs du marché se sont mis à douter de la soutenabilité de la dette de la Grèce et le rendement des obligations grecques a commencé à augmenter. Au début, les dirigeants européens étaient opposés à tout plan de sauvetage pour la Grèce. Cette position se modifie quand il est devenu clair que les inquiétudes des marchés ont commencé à se propager à d'autres pays, tels que le Portugal, l'Espagne, l'Irlande ou l'Italie.

Suivant la recommandation de la Commission européenne, la Grèce a mis en route un plan d'austérité rigoureux en mars 2010, à hauteur de $2 \%$ du PIB (Pisani-Ferry et al., 2013, p. 139). Cependant, il est vite devenu évident que la Grèce serait loin d'atteindre son objectif de déficit. Fin avril 2010, le pays finit par demander l'aide des États membres de la zone euro et du FMI. Début mai 2010, la troïka (FMI, Commission européenne et Banque 
centrale européenne) était constituée et mettait sur pied un plan de sauvetage de 110 milliards d'euros, tout en avertissant que la Grèce avait de sérieux problèmes de solvabilité ne pouvant être résolus sur la seule base des programmes d'ajustement structurel qui y furent associés.

Malgré les importantes coupes budgétaires prévues, l'analyse de la soutenabilité de la dette grecque n'escomptait qu'une récession relativement légère. Le rapport appuyant le programme supposait une réduction du déficit budgétaire, ajustée au cycle, de $10 \% \mathrm{du}$ PIB en 2009 à $2,4 \%$ en 2010 et proche de l'équilibre en 2011. La contraction induite du PIB envisagée était de $4 \%$ en 2010 et $2,6 \%$, en 2011 , devant être suivie d'un retour de la croissance en 2012 (FMI, 2010). Même avec une hypothèse aussi optimiste, le ratio dette publique/ PIB ne devait s'élever qu'à 150 \% du PIB. À ce moment déjà, des universitaires ont exprimé leurs doutes quant à ces hypothèses, déclarant qu'un tel optimisme était complètement irréaliste et que le niveau de la dette pourrait aisément s'avérer bien plus élevé (i.e. Dullien et Schwarzer, 2010).

60 Ainsi que le montrent des documents qui ont fuité dans le Wall Street Journal (2013), plusieurs directeurs exécutifs du FMI ont également exprimé en interne leurs craintes à propos du programme, notamment les représentants de l'Argentine, du Brésil et de l'Inde. En outre, le directeur exécutif suisse René Weber, cité dans une déclaration préparée pour le Conseil du 9 mai 2010, tenait les propos suivants :

[« Nous avons] des doutes considérables sur la faisabilité du programme. [...] Nous avons des doutes sur les hypothèses de croissance, qui semblent être excessivement bienveillantes. Même un petit écart négatif des projections de référence de la croissance rendrait insoutenable le niveau de la dette sur le long terme. [...] Pourquoi une restructuration de la dette ou l'implication du secteur privé dans le plan de sauvetage n'ont-ils pas été pris en compte jusqu'à présent ?"

61 Le plan n'en fut pas moins approuvé par les organes compétents du FMI. Il échouera à restaurer la confiance des investisseurs, la Grèce ne parvenant pas à accéder de nouveau aux marchés financiers. On le sait, l'économie grecque plongera dans une profonde récession, comme le FMI et la troïka l'avaient prévu.

62 Vers la fin de l'année 2010, il est devenu évident que le premier plan de sauvetage grec n'était pas suffisant et, en 2011, ont débuté des discussions concernant un second plan de renflouement. À ce moment dominait le sentiment que les prêts de sauvetage seuls n'étaient pas suffisants alors qu'il était désormais prévu que la dette du gouvernement grec approche bientôt $200 \%$ du PIB. Ainsi, les dirigeants de l'UE ont-ils convenu en octobre 2011 de faire contribuer les créanciers du secteur privé grec, ce qui sera mis en œuvre en 2012, soit à peu près deux ans après la première fourniture de liquidités.

Cependant, la restructuration imposée de la dette n'aura pas eu d'effet significatif sur le niveau de la dette. Premièrement, alors que les institutions de la troïka, dont la Banque centrale européenne, ont alors acheté une quantité importante d'obligations grecques, la dette officielle (auprès des institutions de la Troïka, dont la BCE qui avait acheté un montant substantiel de titres grecs) fut exclue de la restructuration. Deuxièmement, comme la restructuration de la dette a épuisé les fonds propres du secteur bancaire grec, le gouvernement a été contraint de trouver de nouveaux fonds pour procéder à une recapitalisation du secteur bancaire. Selon l'analyse du FMI lui-même (2012a), la restructuration de la dette ne devait faire retomber que légèrement le niveau de la dette grecque, de 165 à 160 \% du PIB. Les services du FMI ont ainsi écrit (2012a, p. 3f) : 
« La nature du fonctionnement du PSI (Policy support instrument ou Participation du secteur privé) [...] avec l'échelonnement de l'aide officielle, [...] augmente significativement la rigidité de la dette grecque, ce qui a des implications sur la capacité de la Grèce à mobiliser de nouveaux financements privés en grandes quantités et dans des termes convenables même après que l'économie s'est stabilisée et que la croissance a reprise. [...] Le fardeau du service de la dette extérieure de la Grèce, surtout d'échéances à court terme, a augmenté et reste lourd. [...]» Déjà au début 2010, les spreads d'autres pays périphériques de la zone euro ont augmenté parallèlement au rendement des obligations grecques, quoique restant à un niveau inférieur (Graphique 1). Juste après le premier plan de sauvetage de la Grèce, les spreads d'autres pays périphériques de la zone euro ont chuté, mais ont recommencé à monter presque immédiatement. Dans le but de rassurer les marchés financiers le gouvernement portugais a annoncé des mesures d'austérité en mai 2010. Avec l'impact de ces mesures, l'économie portugaise est retombée en récession; le PIB ayant commencé à se rétracter au quatrième trimestre de 2010. Par la suite, les finances publiques se sont détériorées et le Portugal s'est vu contraint de faire passer de nouvelles mesures d'austérité, alors qu'il n'avait pas réussi à rassurer les marchés financiers, les spreads sur les obligations portugaises continuant d'augmenter. 


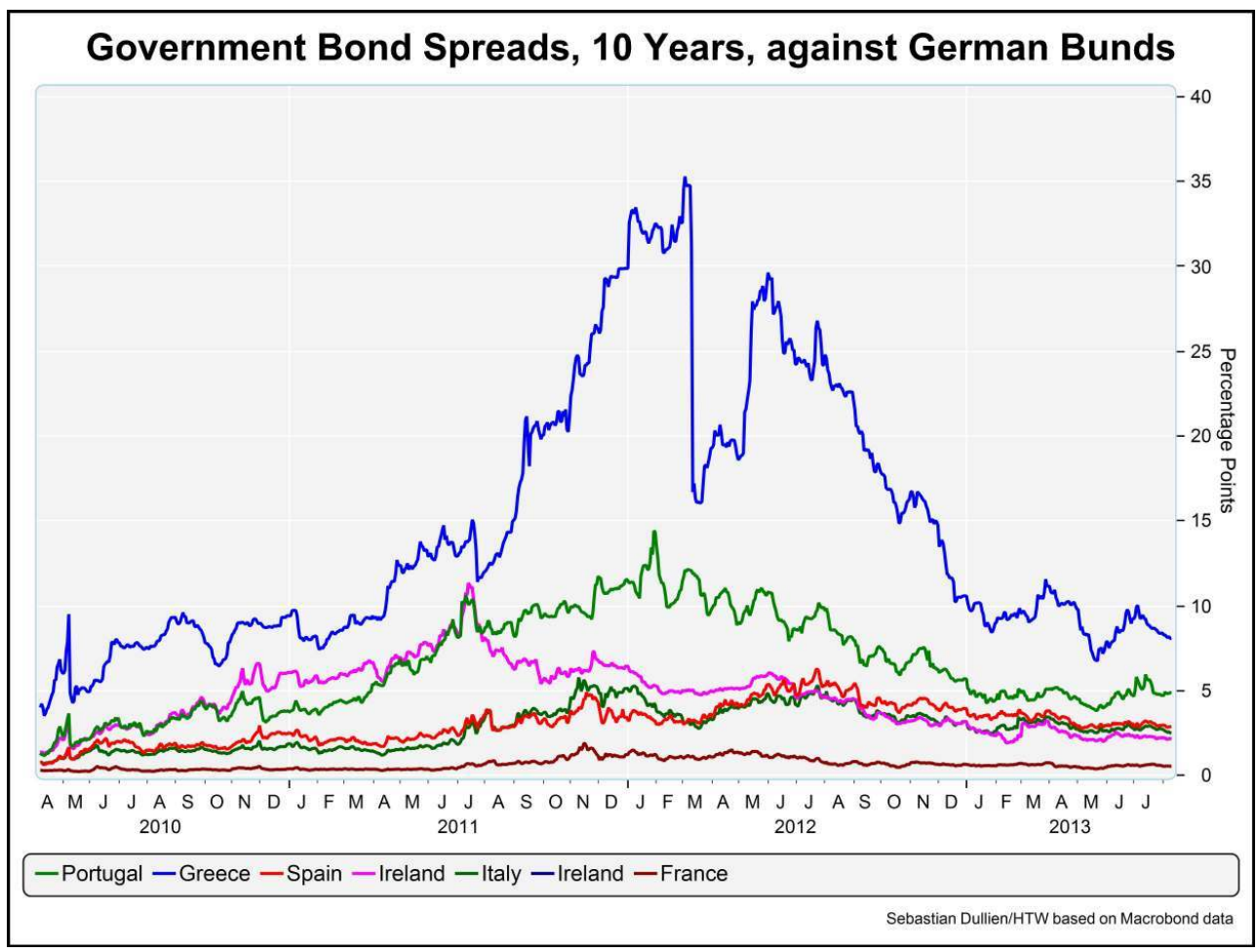

Pendant ce temps, les inquiétudes à propos du système bancaire irlandais se sont aggravées parallèlement à une forte contraction de l'activité économique. Les spreads sont montés à plus de 360 points de base en août 2010. Après l'annonce de coûts de restructuration de plus de 50 milliards d'euros en septembre 2010, le gouvernement s'est vu contraint de proposer un programme d'austérité drastique en octobre 2010 et a finalement sollicité une aide financière de la part du FMI et de ses partenaires européens en décembre 2010. Avec des données économiques et des finances publiques dégradées, le Portugal suivit en avril 2011. Dans les deux cas, les programmes de la troïka ont inclus des mesures d'austérité drastiques, des réductions des dépenses budgétaires et l'augmentation des impôts.

Comme les traités européens contiennent une "clause de non-renflouement " selon laquelle les États-nations sont responsables de leurs propres dettes, les dirigeants européens étaient d'abord réticents à mettre en place de grandes structures de sauvetage permanentes et les premiers plans ont été limités en quantité et en durée. En fait, jusqu'au repli de 2010, les dirigeants européens ne s'étaient pas mis d'accord pour remplacer le Fonds européen de stabilité financière, déclaré comme temporaire, par le Mécanisme européen de stabilité (MES), permanent, lequel serait doté d'une capacité de financement de 500 milliards d'euros et devait être intégré aux traités européens.

Néanmoins, même ces nouveaux pas échoueront à faire baisser efficacement les spreads des obligations de l'Europe dite périphérique. Même les pays qui avaient un ratio dette/ PIB encore relativement modéré, comme l'Espagne (qui avait un ratio dette/PIB d'environ $69 \%$ en 2011, presque 10 points de moins que l'Allemagne) ont vu leurs spreads vis-à-vis des obligations allemandes augmenter au point d'atteindre plus de 500 points de base. On craindra alors que la capacité de financement du MES s'avère trop basse, si l'Italie ou l'Espagne réclamaient de l'aide. Comme l'ont rappelé Giovanni et Gros (2012) en mars 
2012, les programmes pour les (petits) pays, la Grèce, l'Irlande, le Portugal, s'élevaient déjà à des engagements de 490 milliards d'euros, tandis que les besoins de refinancement de l'Italie et de l'Espagne sur la période allant de 2012 à 2016 s'élevaient à presque 1800 milliards d'euros. Ensuite, les spreads sur les obligations périphériques ont encore grimpé sans interruption au long du printemps 2012 et ils dépasseront les 500 points de base pour l'Italie et l'Espagne au cours de l'été.

70 C'est alors que le président de la $\mathrm{BCE}$, Mario Draghi, a prononcé à Londres le fameux discours, largement repris, déclarant que la BCE ferait « ce qu'il faut » pour sauver l'euro. Peu après, la BCE a expliqué ce que cela signifiait dans un premier temps : sous le terme "Opérations monétaires sur titres", elle était disposée à acheter sans limite prédéterminée des obligations gouvernementales de pays soumis à un programme du MES qui risquait de perdre tout accès aux marchés financiers. Cet évènement est désormais largement considéré comme un tournant majeur décisif de la crise de l'euro, puisque les taux d'intérêt sur les obligations périphériques ont alors commencé à chuter et n'ont pas de nouveau fortement augmenté à ce jour.

\subsection{L'évaluation ex-post du FMI et ses positions variables}

Il y a des signes - au moins à huis clos - que la position du FMI au sein de la troïka était l'une des plus flexibles concernant la combinaison entre restructuration de la dette et politiques d'ajustement (voir i. e. Rapport du Parlement Européen [2014]). En ce sens, la position interne du FMI reflète le virage déjà pris par Lütz et Kranke (2014), lequel témoigne de l'importante flexibilité qu'avait démontré le FMI en comparaison aux instances européennes lorsqu'il s'était attaqué aux crises financières de membres emprunteurs, tels que les pays d'Europe de l'Est, avant la crise grecque. Le FMI a indéniablement transmis une connaissance approfondie aux institutions européennes sur la manière de mettre en place des programmes de liquidité. Les institutions européennes ont également pu acquérir un savoir accru dans la compréhension de la nature la crise de l'euro.

«Les ministres des Finances qui débattent dans l'Eurogroupe et les hauts fonctionnaires qui préparent les réunions concèdent de plus en plus qu'influer euxmêmes sur les anticipations du marché peut générer des crises ayant les caractéristiques d'une prophétie auto-réalisatrice [...]. Cette idée a été associée aux modèles à «équilibres multiples", qu'ont utilisés la BCE et le FMI ainsi qu'un certain nombre d'universitaires et de think tankers pour expliquer l'évolution de la crise (Schwarzer, 2015, p. 18). »

On soutiendra toutefois que ce transfert de connaissances a été pour le moins limité. En suivant les acquis du FMI lors des crises latino-américaines, la Grèce n'aurait jamais dû recevoir de soutien sans restructuration de la dette et les autres pays de la zone euro n'auraient pas dû se voir imposer l'austérité drastique qui leur a été imposée. La forme de fourniture de liquidité encouragée par la BCE à partir de 2012 aurait plutôt dû être la réponse apportée depuis le début.

Dans chaque cas, le FMI a assoupli ses critères pendant la crise de l'euro. Assez tôt dans les négociations sur la crise de la dette grecque, le FMI l'a identifiée à juste titre comme un cas d'insolvabilité. EN 2010, la dette du pays était d'ores et déjà classée à " haute probabilité de ne pas être soutenable » (voir FMI, [2013a, p. 18]).

«Le Fonds a approuvé un prêt exceptionnellement important à la Grèce dans le cadre du SBA en mai 2010 malgré des doutes considérables sur la soutenabilité de la 
dette de la Grèce (...). La décision a entrainé le non-respect par le Fonds de ses règlements établis sur l'accès exceptionnel. (...) Les partenaires européens qui avaient écarté une restructuration de la dette étaient réticents à fournir des garanties de financement additionnelles (FMI, 2013a, p. 32). »

74

Malgré cela, le FMI a convenu avec ses partenaires européens de la troïka de fournir des liquidités à la Grèce. Dans le but de rendre cet accord compatible avec son propre règlement, le Fonds a ensuite changé l'EAP en 2010.

« Le cap consistait par conséquent en la modification des politiques pour créer une exception aux conditions nécessaires de «haute probabilité» dans des circonstances où «il $\mathrm{y}$ a un risque élevé de débordements systémiques internationaux ». Finalement, l'ajustement prévu s'est révélé infaisable et, malgré un financement supplémentaire du secteur public comme condition de soutien, la restructuration de la dette privée est devenue inévitable et a été lancée en février 2012 (FMI, 2013b, p. 20).»

Avec cette exception dans le cadre du prêt à la Grèce, malgré de sérieux doutes sur sa solvabilité budgétaire, le FMI s'est clairement contredit dans ses propres approches, remodelées après la gestion des crises des économies émergentes et traduites dans un cadre réglementaire précis. Le FMI lui-même a expliqué son attitude par un ensemble multiple d'intérêts des pays emprunteurs et des pays créanciers pour reporter la restructuration d'une dette insoutenable.

«Les autorités sont également concernées par l'impact d'une restructuration sur le retour de l'accès aux marchés et les effets de contagion sur le secteur privé. En outre, des créanciers institutionnels ont parfois contribué aux reports, par crainte qu'une restructuration diminue la motivation du pays débiteur à ajuster, contraigne les banques situées dans les pays des prêteurs institutionnels à admettre des pertes, provoque l'agitation des marchés et touche des pays en situation analogue, ou pour préserver la flexibilité à l'avenir. Naturellement, les créanciers privés souhaiteront également éviter une restructuration de la dette dans la mesure $\mathrm{du}$ possible, et feront donc pression pour un renflouement par le secteur public. (FMI, 2013b, p. 21).»

En même temps, le FMI a clairement admis que la provision de liquidités en cas d'insolvabilité était beaucoup plus coûteuse qu'une initiative de restructuration directe de la dette : « [...] quand une restructuration de la dette est l'unique option pour gérer un choc de liquidités ou pour rétablir la solvabilité, par exemple dans des situations où le financement disponible et la politique d'ajustement ont été épuisés, les délais finissent par amplifier les coûts finaux. »(FMI, 2013b, p. 20f). Comme le FMI a toujours été remboursé, ces "coûts finaux» peuvent uniquement faire référence aux coûts d'ajustement et à la production perdue dans le pays concerné.

Ce n'est que récemment que le FMI a débattu d'un nouveau cadre règlementaire (FMI, 2013b, 2014) pour répondre aux cas d'illiquidité et d'insolvabilité, faisant ainsi valoir les exceptions octroyées à la Grèce en 2010. Par ailleurs, outre les classifications de crises d'illiquidité et de crises d'insolvabilité, une troisième option a été introduite pour les cas qui ne peuvent pas être clairement classés comme relevant de l'une ou l'autre. Dans de tels cas, une prolongation des échéances sera proposée au pays concerné au lieu de liquidités ou de restructuration de la dette. Dans le cas de ce qu'on appelle un reprofilage fructueux, le Fonds soutient que la perte du créancier est moindre que dans le cas d'une restructuration. Dans le cas où la crise empire, une restructuration devrait être menée. Néanmoins, le concept d'équilibres multiples ainsi que l'expérience du FMI en Argentine et en Grèce montrent qu'une telle solution est associée à des coûts plus élevés 
et à une crise prolongée si une prolongation des échéances n'aboutit pas. La restructuration postérieure est bien plus coûteuse pour les deux parties et rend possible, pour les créanciers, le report la restructuration.

Outre le cas de la Grèce, il faut rappeler que le montant des approvisionnements de liquidités à d'autres pays de la zone euro a été considéré comme insuffisant et divers signes montrent que le FMI en était conscient. On peut citer, par exemple, le World Economic Outlook de septembre 2011 (FMI, 2011, p. 19), soit à peu près 10 mois avant le discours de Mario Draghi, où le FMI a déclaré :

" [Le défi consiste à envoyer] le signal fort que les membres de la zone euro vont continuer de maintenir leur confiance en l'euro quoi qu'il en coûte. Entre-temps, la BCE aura besoin de continuer à intervenir énergiquement avec des garanties souveraines appropriées qui soutiennent un fonctionnement ordonné des marchés d'obligations publiques. »

Le 18 juillet 2012, environ une semaine avant le discours de Draghi, une enquête online du FMI relaye l'appel suivant de Mahmoud Pradhan, Directeur adjoint du Département Europe du FMI et chef de mission pour la zone euro (FMI, 2012b) : «La Banque centrale européenne devrait, de notre point de vue, envisager des mesures moins conventionnelles (par exemple, l'assouplissement quantitatif) pour soutenir les marchés financiers dans les pays subissant une tension intense. »

Il semble donc que, tout en étant contraint par ses propres restrictions de prêt, le FMI essayait désespérément de pousser ses partenaires de la troïka à fournir plus de liquidités par des moyens allant au-delà des programmes de prêts déjà établis.

\section{Conclusion}

81 Au regard du référentiel théorique décrit ci-dessus, les modèles à équilibres multiples qui engageaient un traitement différencié des crises de la dette en fonction de l'état de solvabilité du débiteur, la chaine des événements de la crise de l'euro est plutôt simple à interpréter : en Grèce, il semble qu'un problème d'insoutenabilité de la dette, constaté dès le début de la crise, aurait nécessité une restructuration de la dette immédiate et satisfaisante, ainsi que des mesures de redressement budgétaire. Les provisions de liquidités de la troïka ont seulement abouti à retarder la restructuration de la dette et à en augmenter potentiellement les coûts. Dans le reste de la zone euro, l'élément important réside dans le fait qu'il y ait eu des problèmes d'illiquidité plutôt que d'insolvabilité, comme l'a prouvée la fin de la panique dès l'annonce d'une offre illimitée de liquidités de la part de la BCE et du MES. Ici, le problème semble résider dans le fait que, jusqu'à l'annonce de Mario Draghi, le volume de l'aide possible en liquidités était vraisemblablement insuffisant. Comme le décrivent les modèles à équilibres multiples, seul un montant approprié de liquidités, disponible de manière crédible, parvient à stabiliser les anticipations d'un équilibre sans défaut (« le bon équilibre »). En revanche, un montant insuffisant de liquidités ne pourra pas modifier les anticipations et le pays demeurera soumis au risque de défaut. Plus l'offre de liquidités est retardée, plus le défaut devient plausible, étant donné que les taux d'intérêt élevés dégradent la position solvable sous-jacente du pays en question.

82 Ces leçons théoriques, conjointement à l'expérience pratique douloureuse, acquise en s'attaquant aux crises de la dette des économies émergentes, ont été prises en compte dans le cadre réglementaire du FMI. Aussi, peut-on dire que les espoirs des Européens de 
bénéficier de plusieurs décennies d'expérience de gestion des crises par le FMI n'ont été que partiellement comblés, et ceci, dans des délais longs et coûteux. Les connaissances fondamentales dans la conception des plans d'aide ont été particulièrement négligées, en dépit de l'évidence que les services du FMI étaient bien conscients que la conception des programmes de la troïka pour la zone euro s'opposait aux acquis de son savoir institutionnel. Comme les choix politiques pris ont en fait ignoré les expériences du FMI et ont sans doute contribué à l'approfondissement et à la longue durée de la crise de l'euro, cet échec pointe un sérieux défaut de l'organisation de la troïka. Nous confirmons donc la conclusion de Ban et Gallagher (2015) comme quoi ces changements sont plutôt lents et inégaux.

83 Cela soulève un certain nombre de questions pour la recherche future. Il est important de se demander pourquoi le FMI n'a pas davantage mis en avant dans la crise de l'euro les nouvelles approches mises en place après les expériences des économies émergentes. Était-ce parce qu'il n'était qu'un « partenaire mineur » des programmes de la troïka, dont la part la plus grande de fonds déboursés venait de sources européennes? Sinon, était-ce dû à l'économie politique des structures de gouvernance du FMI et au fait que les pays de l'UE sont parmi les plus importants actionnaires du Fonds? Ce genre de questions nécessiterait des connaissances approfondies des processus de prise de décision formels et informels au sein du FMI, de la troïka et des gouvernements impliqués, qui outrepassent le cadre de cet article.

En fonction des réponses à ces questions, d'importantes conclusions politiques pourraient être tirées sur la future organisation des programmes d'aide et sur le FMI. Si c'est l'influence européenne au sein du Fonds qui a conduit à ces conceptions politiques qu'il faut qualifier de sous-optimales, cela appellerait à des réformes dans la structure de gouvernance du FMI. Si le fait est plutôt attribuable à une influence injustifiée des partenaires principaux de la troïka, alors le FMI pourrait envisager de s'abstenir de rejoindre de tels programmes d'aide.

Néanmoins, nous pouvons conclure que, en utilisant mieux son propre savoir accumulé et formalisé sur le plan institutionnel, le FMI aurait dû réussir à aider les membres la zone euro à s'attaquer à cette crise, ce qui aurait probablement fait la différence. En se reportant à la déclaration d'un ancien directeur de la BCE concernant le rôle assigné au FMI au sein de la troïka, qu'on a citée en introduction, on peut dire que le FMI a mieux réussi dans le second aspect, à savoir le rôle attribué de gendarme externe, plutôt que l'apport de son expérience propre.

BIBLIOGRAPHIE

Asmussen J. (2012), Griechenland ist keine Bedrohung für die Welt, Interview in Financial Times Deutschland, $20 \mathrm{Feb}: 17$.

Bagehot W. (1873), Lombard Street: A Description of the Money Market, London, Henry S. King and Co. 
Ban C. et K. Gallagher, (2015), "Recalibrating Policy Orthodoxy: The IMF Since the Great Recession," Governance, 28 (2), pp. 131-146.

Blanchard O., G. Dell'Ariccia, et P. Mauro, (2010), Rethinking Macroeconomic Policy, SPN/10/03, Washington DC, IMF.

Blanchard O. (2012), "Monetary Policy in the Wake of the Crisis," in O. Blanchard et al. (eds.), In the Wake of the Crisis, Cambridge/London, MIT Press, pp. 7-13.

Chang R. et A. Velasco, (2000), "Financial Fragility and the Exchange Rate Regime," Journal of Economic Theory, 92 (1), pp. 1-34.

Cole H. et T. Kehoe, (1996), “A self-fulfilling model of Mexico's 1994-1995 debt crisis,” Journal of International Economics, 41 (3-4), pp. 309-330.

Corsetti G., P. Pesenti et N. Roubini, (1998), Paper Tigers ? A Model for the Asian Crisis, NBER Working Paper $N^{\circ} 6783$, Washington DC, NBER.

Damill M., R. Frenkel et M. Rapetti, (2012a), The Argentine Foreign Debt Default and Restructuring, Itf Policy Brief 63, Buenos Aires, ITF. URL:

http://www.itf.org.ar/pdf/lecturas/lectura63.pdf.

Damill M., R. Frenkel et M. Rapetti, (2012b), Fiscal Austerity in a Financial Trap: The Agonic Years of the Convertibility Regime in Argentina, ITF Policy Brief 62, Buenos Aires, ITF. URL: http:// www.itf.org.ar/pdf/lecturas/lectura62.pdf.

DeMello L. et D. Moccero, (2006). Brazil's Fiscal Stanceduring 1995-2005: The Effect of Indebtedness on Fiscal Policy Over the Business Cycle, OECD Economics Department Working Papers $\mathrm{N}^{\circ} 485$, Paris, OECD. URL:

http://dx.doi.org/10.1787/857281763217.

De Vries M. (1987), Balance of Payments Adjustment, 1945 to 1986. The IMF Experience, Washington, IMF.

Dullien S. et D. Schwarzer, (2010), Umgang mit Staatsbankrotten in der Eurozone [Dealing with State Bankruptcies in the Eurozone], Stiftung Wissenschaft und Politik. URL :

http://www.swp-berlin.org/fileadmin/contents/products/studien/2010_S19_dll_swd_ks.pdf

Eichengreen B. (2002), A Temporary Respite for Brazil. URL:

http://eml.berkeley.edu/ eichengr/reviews/swissreview10sep3-02.pdf.

Eichengreen B. et R. Hausmann, (2005), Other People's Money - Debt Denomination and Financial Instability in Emerging Market Economies, Chicago, University of Chicago Press.

Eichengreen B. et al., (2011), Rethinking Central Banking, Committee on International Economic Policy and Reform, Washington DC, Brookings Institution.

European Parliament (2014), On the enquiry on the role and operations of the Troika (ECB, Commission and IMF) with regard to the euro area programme countries, EP Document 2013/2277(INI), Committee on Economic and Monetary Affairs (Rapporteur: Othmar Karas, Liem Hoang Ngoc), 28 February, accessed at http://www.europarl.europa.eu/sides/getDoc.do? type $=$ REPORT\&mode $=X M L \&$ reference=A7-2014-0149\&language $=\mathrm{EN}_{2}$, September 2014 .

“EU-IMF feud erupts over Greek debt," (2012), Financial Times, November 12.

Flood R. et N. Marion, (1996), Policy Implications of "Second-Generation" Crisis Models, IMF Working Paper 97/16, Washington DC, IMF. 
Fratzscher M. (2013), Es gibt keine Euro-Krise [There is no Euro Crisis], DIW-Wochenbericht, $\mathrm{N}^{\circ}$ 15/2013, Berlin, DIW.

Giovannini A. et D. Gros, (2012), How high the firewall? Potential financing needs for the periphery, CEPS Commentary, Brussels, CEPS.

Holland D. et J. Portes, (2012), “Self-defeating austerity?" in National Institute Economic Review, 222 (1), pp. F4-F10.

"IMF Document Excerpts: Disagreements Revealed," (2013), Wall Street Journal, October 7. URL : http://blogs.wsj.com/economics/2013/10/07/imf-document-excerpts-disagreements-revealed/? mod=wsj_streaming_stream.

IMF (2000a), Argentina: Memorandum of Economic Policies, Washington DC, IMF. URL: http://www.imf.org/external/np/loi/2000/arg/01/index.htm.

IMF (2000b), Argentina: Argentina Letter of Intent, Memorandum of Economic Policies, and Technical Memorandum of Understanding, Buenos Aires, IMF. URL: http://www.imf.org/external/np/loi/2000/arg/03/index.htm.

IMF (2002a), Assessing Sustainability, Prepared by the Policy Development and Review Department, in consultation with the Fiscal Affairs, International Capital Markets, Monetary and Exchange Rate Affairs, and Research Departments, Washington DC, IMF. URL:http:// www.imf.org/external/np/pdr/sus/2002/eng/052802.pdf.

IMF (2002b), Brazil-Letter of Intent, Memorandum of Economic Policies, and Technical Memorandum of Understanding, Brasília, IMF. URL : http://www.imf.org/external/np/loi/2002/bra/04/index.htm.

IMF (2003), Access Policy in Capital Account Crises - Modifications to the Supplemental Reserve Facility (SRF) and Follow-up Issues Related to Exceptional Access Policy, Policy Development and Review and Treasurer's Departments, Washington DC, IMF. URL: https://www.imf.org/external/np/tre/access/2003/011403.htm.

IMF (2004a), The IMF and Argentina, 1991-2001, Washington DC, IMF/Independent Evaluation office.

IMF (2010), Greece: Staff Report on Request for Stand-By Arrangement, IMF Country Report N ${ }^{\circ}$ 10/110, May 2010, Washington DC, IMF. URL: http://www.imf.org/external/pubs/ft/scr/2010/cr10110.pdf

IMF (2011), World Economic Outlook: Slowing Growth, Rising Risk, September 2011, Washington DC, IMF URL:

http://www.imf.org/external/pubs/ft/weo/2011/02/pdf/text.pdf

IMF (2012a), Greece-Assessment of the Risks to the Fund and the Fund's Liquidity Position, March 9, 2012, Appendix to the country report, Washington DC, IMF. URL: http://www.imf.org/external/pubs/ft/scr/2012/cr1257.pdf

IMF (2012b), IMF Calls on Eurozone to Take Determined Action in Response to Crisis, IMF Survey Online, July 18, 2012, Washington DC, IMF. URL:

http://www.imf.org/external/pubs/ft/survey/so/2012/int071812a.htm

IMF (2013a), Greece: Ex Post Evaluation of Exceptional Access under the 2010 Stand-By Arrangement, IMF Country Report $\mathrm{N}^{\circ} 13 / 156$, Washington DC, IMF. URL: http://www.imf.org/external/pubs/ft/scr/2013/cr13156.pdf 
IMF (2013b), Sovereign Debt Restructuring - Recent Developments and Implications for the Fund's Legal and Policy Framework, Washington DC, IMF. URL: https://www.imf.org/external/np/pp/eng/2013/042613.pdf

IMF (2014), The Fund's Lending Framework and Sovereign Debt - Preliminary Considerations, Washington DC, IMF.

Kaminsky G. et C. Reinhart, (1999), “The Twin Crises: The Causes of Banking and Balance-ofPayments Problems," American Economic Review, 89 (1), pp. 473-500.

Krampf A. (2015), "Perhaps this time it's different: ideas and interests in shaping international responses to financial crises," Contemporary Politics, 21 (2), pp. 179-200.

Köhler H. (2000), Address by Horst Köhler, Chairman of the Executive Board and Managing Director of the International Monetary Fund, to the Board of Governors of the Fund, Prague, September 26, 2000.

http://www.imf.org/external/np/speeches/2000/092600.htm.

Krugman P. (1979), “A model of balance of payments crises," Journal of Money, Credit and Banking, 11 (1), pp. 311-25.

Krugman P. (1999), “Currency Crises,” in M. Feldstein (eds.), International Capital Flows, Chicago, University of Chicago Press, pp. 421-440.

Krugman P. (2003), “Crisis - The Next Generation,” in E. Helpman et E. Sadka (eds.), Economic Policy in the International Economy: Essays in Honor of Assaf Razin, Cambridge, Cambridge University Press, pp. 15-33.

Laeven L. et F. Valencia, (2012), Systemic Banking Crises Database: An Update, Working Paper 12/163, Washington DC, IMF.

Larsen F. (2003), Argentina and the IMF - The Need for Perspective, Address by the Director, Offices in Europe, of the International Monetary Fund, Toulouse, November 18-22. URL: http://www.imf.org/external/np/speeches/2003/111803.htm.

Lütz S. et M. Kranke, (2014), The European rescue of the Washington Consensus? EU and IMF lending to Central and Eastern European countries, Review of International Political Economy. URL: http://www.tandfonline.com/doi/full/10.1080/09692290.2012.747104

Masson P. (1999), Multiple Equilibria, Contagion, and the Emerging Market Crises, IMF Working Paper 99/164, Washington DC, IMF. URL:

http://www.imf.org/external/pubs/ft/wp/1999/wp99164.pdf.

Ministério da Fazenda do Brasil (2003), Política econômica e reformas estruturais [Economic Policy and Structural Reforms], Brasília.

Mussa M. (2002), Argentina and the Fund - from triumph to tragedy, Washington DC, Institute for International Economics.

Obstfeld M. (1996), "Models of Currency Crises with Self-fulfilling Features," European Economic Review, 40 (1), pp. 1037-1047.

Pisani-Ferry J., A. Sapir et A. Wolff, (2013), EU-IMF assistance to euro-area countries: an early assessment, Brussels, Bruegel Blueprint Series.

Rogoff K. (2006), “Who Needs the IMF?” Newsweek International, 25 September. http://www.brookings.edu/research/opinions/2006/09/25macroeconomics-rogoff. Schmieding H. (2014) "Draghi two years on: the words that fixed the euro," Berenberg Macro Views, 23 July. 
Schwarzer D. (2015), "Building the euro area's debt crisis management capacity with the IMF," Review of International Political Economy. DOI: 10.1080/09692290.2014.965263

Shambaugh J. (2012), “The Euro's three crises,” Brookings Papers on Economic Activity, 44 (1), pp. 157-231.

Silva L. da (2002), Carta ao povo brasileiro [Letter to the Brazilian People], 22 June. URL:

http://www.fpabramo.org.br/sala_leitura/carta_povobrasileiro.htm.

Stiglitz J. (2002), Globalization and its discontents, New York, Norton.

\section{NOTES}

1. Citation originale: «Es war richtig, den IWF zu beteiligen. Der Fonds hat eine einmalige Erfahrung im Entwerfen solcher Programme. Er ist zudem wie ein externer Polizist in Europa, der weniger Rücksichten nehmen muss. » (Entretien dans le Financial Times Deutschland du 20 février 2012).

2. Une minorité de créanciers institutionnels a refusé cet accord et s'est pourvu en appel devant la justice américaine. Celle-ci a statué en 2014 que l'Argentine devait s'acquitter de ses obligations, décision qui a entraîné la rétention du remboursement de sa dette aux autres créanciers qui avaient accepté le plan de restructuration. En réaction à la décision du tribunal, les agences de notation internationales ont de nouveau déclaré l'Argentine en faillite, ce qui s'est répercuté sur la qualité des obligations.

3. «Premissa dessa transição será naturalmente o respeito aos contratos e obrigações do pais » (Silva, 2002, p. 3).

4. Cependant, la décision de se passer d'un moratoire et d'une restructuration de la dette a eu un prix pour le Brésil. Alors qu'il était au pouvoir en 2003, le nouveau gouvernement de Lula, a non seulement été à la hauteur des attentes quant à son engagement de remplir ses obligations, mais il a également adopté une politique économique ultra-orthodoxe pour maintenir les flux de capitaux. Le nouveau gouvernement a poursuivi les politiques procycliques antérieures et a fait monter l'excédent budgétaire primaire à 4,25\%, au-dessus de l'objectif du FMI (Ministério da Fazenda do Brasil, 2003, p.9). Il a maintenu cette politique les années suivantes et les taux d'intérêt réel bien au-dessus de $10 \%$. En conséquence, l'économie brésilienne est entrée en récession en 2003, et n'a recommencé à croître lentement qu'en 2004, en même temps que la flambée mondiale du cours des matières premières.

\section{RÉSUMÉS}

Cet article s'interroge sur le fait de savoir si l'expérience acquise par le FMI lors des crises des dettes souveraines des économies émergentes a été utile pour gérer la crise de l'euro. Nous faisons le lien entre les modèles d'équilibres multiples et l'expérience acquise par le FMI au cours des crises latino-américaines des années 2000. Nous analysons ensuite les changements qui en ont découlé quant aux lignes directrices des politiques menées par le FMI. Nous montrons que les connaissances antérieures ont été prises en compte, mais appliquées tardivement et, dans le cas de la Grèce, en partie à l'encontre des règles institutionnelles ou des recommandations internes. 
En ce qui concerne la Grèce, bien que le FMI ait plaidé - au moins en interne - pour une restructuration rapide de la dette, l'institution y a associé plusieurs programmes d'émission de liquidité. Par conséquent, nous soutenons que, dans le cadre de la lutte contre la crise européenne, le concours du FMI n'a pas tout à fait apporté ce qu'on en pouvait en attendre

The paper asks whether it has been worth bringing in the IMF's experience gained in emerging market sovereign debt crises to deal with the euro crisis. We link models of multiple equilibria with the IMF's experience made in Latin American crises in the 2000s. Further, we analyze subsequent changes in the IMF's policy guidelines and show that previous insights have been taken in, but applied only with significant delay and partially against institutional rules and internal advice for the case of Greece. Even if at least internally the IMF argued for a quick debt restructuring for the case of Greece, the institution joined several liquidity assistance programs. Hence, we argue that the inclusion of the IMF in Europe's crisis fighting approach did not completely deliver what had been hoped for.

\section{INDEX}

Mots-clés : crise de l'euro, crise de la dette des économies émergentes, FMI (Fonds monétaire international), équilibres multiples, mécanisme de résolution de la dette souverain Keywords : euro crisis, emerging market debt crisis, IMF (International Monetary Fund), multiple equilibria, sovereign debt, resolution mechanisms

Code JEL E02 - Institutions and the Macroeconomy, E52 - Monetary Policy, E58 - Central Bank and Their Policies, F53 - International Agreements and Observance $\bullet$ International Organizations

\section{AUTEURS}

\section{BARBARA FRITZ}

Barbara Fritz (auteur principal) est professeur d'économie à l'Institut des études latinoaméricaines et au département d'économie de la Freie Universität Berlin. Institut d'Études latinoaméricaines, Ruedesheimer Str. 54-56, D-14197 Berlin, tel. +49 (0)30 838 53063, barbara.fritz[at]fuberlin.de

\section{SEBASTIAN DULLIEN}

Sebastian Dullien est professeur d'économie internationale à HTW Berlin - Université des sciences appliquées - et membre senior du Conseil européen des relations internationales (ECFR). Hochschule Technik und Wirtschaft Berlin - Université des Sciences appliquées, Fachbereich 3 Wirtschaftswissenschaften, Treskowallee 8, D-10313 Berlin, tel. +49 (0)30 50192547 sebastian.dullien[at]htw-berlin.de

\section{LAURISSA MÜHLICH}

Laurissa Mühlich, Institut d'Études latino-américaines, Ruedesheimer Str. 54-56, D-14197 Berlin, laurissa.muehlich[at]fu-berlin.de 